Pacific Journal of Mathematics

ASYMPTOTIC STABILITY AND NONEXISTENCE OF GLOBAL
SOLUTION FOR A SEMIUINEAR PARABOLIC EQUATION 


\title{
ASYMPTOTIC STABILITY AND NON-EXISTENCE OF GLOBAL SOLUTION FOR A SEMI-LINEAR PARABOLIC EQUATION
}

\author{
C. V. PAO
}

The aim of this paper is to investigate the existence and nonexistence of a nonnegative global solution for a semilinear parabolic system in a bounded domain. It is shown that for a certain class of initial functions the corresponding solution of the initial boundary value problem has a finite escape time, while for another class of initial functions a unique solution exists for all time and diminishes to zero. This result leads to an explicit estimate for the stability and the instability regions of the trivial steady-state solution. In the case of nonexistence of global solutions, an estimate for the finite escape time is also given.

I. Introduction. The problem of nonexistence of global solution for the semilinear parablic equation

$$
u_{t}-\nabla \cdot(D(x) \nabla u)=a u^{1+\alpha}(t \in(0, T\rfloor, x \in \Omega)
$$

has been given considerable attention in recent years (cf. [4-6, 13, 15]). In most of the above references the spatial domain $\Omega$ was taken as the whole space $R^{n}$ so that the system under consideration by these authors is a Cauchy problem. In this paper, we consider a bounded domain $\Omega$ in $R^{n}$ together with the following boundary and initial conditions:

$$
\begin{gathered}
\beta \frac{\partial u}{\partial \nu}+u=0 \quad(t \in(0, T], x \in \partial \Omega) \\
u(0, x)=u_{0}(x) \quad(x \in \Omega) .
\end{gathered}
$$

In the above system, $\nabla$ is the gradient operator, $D$ is a positive function on $\bar{\Omega} \equiv \Omega \cup \partial \Omega, \alpha, \alpha, \beta$ are constants with $\alpha>0, \beta \geqq 0, \partial \Omega$ is the boundary of $\Omega$ and $\nu$ is the outward normal vector on $\partial \Omega$. This system arises from models of simultaneous diffusion and recombination of electrons and irons as well as models of reactor dynamics where positive feedback is allowed when $a>0$ (cf. $[1,8,15]$ ). We assume that $\Omega$ is sufficiently smooth, $D$ is continuously differentiable in $\bar{\Omega}$ and $u_{0}$ is continuous nonnegative on $\bar{\Omega}$ and satisfies the boundary condition (1.2). The constant, $a$, may assume positive or negative values and its magnitude plays an important role in relation to the stability region of the trivial steady state solution. 
The nonexistence of global solution to the Cauchy problem (1.1) and (1.3) for the case $a=D=1$ in the whole space $R^{n}$ has been discussed by Fujita [4, 5], Hayakawa [6], Sugitani [16] and more recently by Portnoy [13]. A similar problem for a bounded domain $\Omega$ has been investigated by Kaplan [7], Friedman [2] and Pao [11]. (See also Levine and Payne [9].) The purpose of this paper is to investigate the existence and nonexistence of a nonnegative global solution to the system (1.1)-(1.3) and to give a threshold result on the asymptotic behavior of the solution. Specifically, we show that for a certain class of initial functions $u_{0}$ a unique nonnegative solution $u(t, x)$ to (1.1)-(1.3) exists on some finite interval $\left[0, T_{0}\right)$ and

$$
\lim \left(\max _{x} u(t, x)\right)=\infty \quad \text { as } \quad t \longrightarrow T_{0} ;
$$

and for another class of initial functions, the solution $u$ exists on $[0, \infty)$ and $\lim u(t, x)=0$ on $\bar{\Omega}$ as $t \rightarrow \infty$. Moreover, we establish in the first case an explicit upper bound for the finite escape time $T_{0}$ while in the second case we give an estimate for the stability region as well as the instability region of the zero solution. We also show that when $a \leqq 0$ the nonnegative solution $u$ diminishes to zero for every nonnegative initial function $u_{0}$. Thus if we increase the value of a from negative to positive then the zero steady state solution changes form global asymptotic stability to regional asymptotic stability; and for initial functions outside certain region the corresponding solutions diverge to infinite in finite time. As is to be expected our estimate demonstrates that larger initial function $u_{0}$ requires less time for the blowing-up property of the solution. Hence the consideration of a bounded domain instead of the whole space $R^{n}$ leads to more delicate asymptotic behavior of the solution. It is interesting to note that this qualitative behavior also has a simple and natural physical interpretation and the results can be extended to more general systems (see the remarks in $\S 2$ ). In the present paper, however, we limit our discussion to the system (1.1)-(1.3).

2. The main results. In order to obtain our main results on the existence and nonexistence of a global solution we as in $[10,11]$ use the notion of upper and lower solutions for parabolic systems. By an upper solution we mean a smooth function $\widetilde{u}(t, x)$ satisfying the inequalities

$$
\begin{array}{ll}
\tilde{u}_{t}-\nabla \cdot(D(x) \nabla \tilde{u}) \geqq \alpha \tilde{u}^{1+\alpha} & (t \in(0, T], x \in \Omega) \\
\beta \frac{\partial \tilde{u}}{\partial \nu}+\widetilde{u} \geqq 0 & (t \in(0, T], x \in \partial \Omega), \\
\widetilde{u}(0, x) \geqq u_{0}(x) & (x \in \Omega)
\end{array}
$$


and a lower solution is a smooth function $\boldsymbol{u}(t, x)$ which satisfies all the reversed inequalities in (2.1). Moreover we require that $\boldsymbol{u} \leqq \tilde{u}$ on $[0, T] \times \bar{\Omega}$. Here by a smooth function it meant a continuous function $w$ on $[0, T] \times \bar{\Omega}$ which is continuously differentiable in $t \epsilon$ $(0, T]$, twice continuously differentiable in $x \in \Omega$ and $\partial u / \partial \nu$ exists on $(0, T] \times \partial \Omega$.

Suppose that upper and lower solutions $\boldsymbol{u}, \widetilde{u}$ exist and $\boldsymbol{u} \leqq \widetilde{u}$. Then by starting from the initial iteration $u^{(0)} \equiv \widetilde{u}$ and $u^{(0)} \equiv \boldsymbol{u}$, respectively, we can construct two sequences from the recurence relation

$$
\begin{array}{ll}
u_{t}^{(k)}-\Gamma\left(D(x) \Gamma u^{(k)}\right)=a\left(u^{(k-1)}\right)^{1+\alpha} & (t \in(0, T], x \in \Omega) \\
\beta \frac{\partial u^{(k)}}{\partial \nu}+u^{(k)}=0 & (t \in(0, T], x \in \partial \Omega) \\
u^{(k)}(0, x)=u_{0}(x) & (x \in \Omega) \\
& \quad k=1,2, \cdots .
\end{array}
$$

Denote these two sequences, respectively, by $\left\{\bar{u}^{(k)}\right\}$ and $\left\{u^{(k)}\right\}$ (so that $\left.\bar{u}^{(0)}=\widetilde{u}, \underline{u}^{(0)}=\boldsymbol{u}\right)$. Then by the properties of upper and lower solutions one can easily show that (i) $\bar{u}^{(k+1)} \leqq \bar{u}^{(k)}$, (ii) $\underline{u}^{(k)} \leqq \underline{u}^{(k+1)}$ and (iii) $\underline{u}^{(k)} \leqq \bar{u}^{(k)}$ for every $k=0,1,2, \cdots$. These inequalities imply that the pointwise limits

$$
\lim \bar{u}^{(k)}=\bar{u} \text { and } \lim \underline{u}^{(k)}=\underline{u} \quad \text { as } \quad k \longrightarrow \infty
$$

exist and satisfy the relation $\boldsymbol{u} \leqq \underline{u} \leqq \bar{u} \leqq \widetilde{u}$ on $[0, T] \times \bar{\Omega}$. In fact, $\underline{u}$ coincides with $\bar{u}$ and is the unique solution of (1.1)-(1.3) (cf. $[10,12])$. Therefore if we can find a pair of upper and lower solution with $\boldsymbol{u} \leqq \tilde{u}$, then a unique solution $u(t, x)$ to (1.1)-(1.3) exists and

$$
\boldsymbol{u}(t, x) \leqq u(t, x) \leqq \tilde{\boldsymbol{u}}(t, x) \text { on }[0, T] \times \bar{\Omega} .
$$

Since for $u_{0} \geqq 0$ the function $\boldsymbol{u}=0$ satisfies all the reversed inequalities in (2.1), the existence of a nonnegative solution $u$ satisfying $0 \leqq u(t, x) \leqq \widetilde{u}(t, x)$ is insured if one can find an upper solution $\widetilde{u}$ which is nonnegative. We shall do this for our global existence problem.

In the proof of the theorems the construction of $\widetilde{u}$ is based on the linear eigenvalue problem

$$
\begin{array}{ll}
\nabla \cdot(D(x) \nabla \dot{\phi})+\lambda \dot{\phi}=0 & (x \in \Omega) \\
\beta \frac{\partial \dot{\phi}}{\partial \nu}+\dot{\phi}=0 & (x \in \partial \bar{\Omega}) .
\end{array}
$$

It is well-known that the least eigenvalue $\lambda_{0}$ is positive and its corresponding eigenfunction $\psi(x)$ is positive in $\Omega$. In fact, if $\beta>0$ the 
maximum principle implies that $\psi(x)>0$ on $\bar{\Omega}$. We normalize is so that $\max \psi(x)=1$. For convenience we set $\psi_{m}=\min \psi(x)$. Notice that $\psi_{m}>0$ when $\beta>0$. Our main results are stated in the following two theorems.

THEOREM 1. Let $\beta>0, a>0$ and $\eta \equiv\left(\lambda_{0} / a\right)^{1 / \alpha} \psi_{m}^{-1}$. Then for any $u_{0}(x) \geqq b \psi(x)$ with $b>\eta$ there exists a constant $T_{0}<\infty$ such that $a$ unique solution $u(t, x)$ to (1.1)-(1.3) exists on $\left[0, T_{0}\right) \times \bar{\Omega}$ and satisfies

$$
\lim _{t \rightarrow T_{0}^{\prime}} \max _{x \in \bar{\Omega}} u(t, x)=\infty \text {. }
$$

Moreover,

$$
T_{0} \leqq\left(\alpha \lambda_{0}\right)^{-1} \ln \left[a\left(b \psi_{m}\right)^{\alpha} /\left(a\left(b \psi_{m}\right)^{\alpha}-\lambda_{0}\right)\right]
$$

THEOREM 2. Let $\lambda \in\left(0, \lambda_{0}\right)$ and $\rho_{\lambda}=\left(\left(\lambda_{0}-\lambda\right) / a\right)^{1 / \alpha}$ when $a>0$. Then for any $u_{0}(x) \leqq \rho_{\lambda} \psi(x)$, a unique nonnegative solution $u(t, x)$ to (1.1)-(1.3) exists on $[0, \infty) \times \bar{\Omega}$ and satisfies

$$
0 \leqq u(t, x) \leqq \rho_{\lambda} e^{-\lambda t} \psi(x) \quad(t>0, x \in \bar{\Omega}) .
$$

In the case of $a \leqq 0$ the above inequality holds for every $\rho_{\lambda} \equiv$ $\rho<\infty$ whenever $u_{0}(x) \leqq \rho \psi(x)$.

REMARK. (a) The results in Theorems 1 and 2 show that when $a>0$ a stability region of the zero solution is given by the set $\Lambda_{1} \equiv$ $\left\{u_{0} ; 0 \leqq u_{0} \leqq \rho_{\lambda} \psi\right\}$ while an instability region is $\Lambda_{2} \equiv\left\{u_{0} ; u_{0} \geqq b \psi\right\}$. By choosing $\lambda$ sufficiently small the value of $\rho_{\lambda}$ may be taken arbitrarily close to $\left(\lambda_{0} / a\right)^{1 / \alpha}$. Since $\lambda_{0}$ is directly related to the size (for a fixed geometry) of the domain $\Omega$ it follows that smaller domain tends to stabilize the equilibrium solution $u=0$ while large domain tends to destabilize the zero solution. This is, of course, to be expected physically since energy leaks faster in smaller domains than larger ones.

(b) Theorems 1 and 2 remain true when the operator $\nabla \cdot(D(x) \nabla u)$ is replaced by a more general uniformly elliptic operator in the form

$$
L u \equiv \sum_{i=1}^{n} a_{i j}(x) u_{x_{i} x_{j}}+\sum_{i=1}^{n} b_{i}(x) u_{x_{i}} .
$$

In this situation the least eigenvalue $\lambda_{0}$ and its corresponding eigenfunction $\psi$ should be with respect to the operator $L$ (under the same boundary condition). Notice that $\lambda_{0}>0$ (or at least there exists a eigenvalue $\lambda_{0}$ which is real positive) and the corresponding eigenfunction $\psi$ is positive (cf. [14]).

The results in Theorems 1 and 2 can be given a physical inter- 
pretation such as in the process of diffusion or heat conduction. When the spatial domain is bounded, energy (or heat) leaks through the boundary surface $\partial \Omega$. Thus for the class of initial functions $u_{0} \leqq \rho_{\lambda} \psi$, energy leaks away through the boundary surface before it gets large so that the effect due to the feedback term $a u^{1+\alpha}$ (which acts as a source for $a>0$ ) is diminishing. But when the initial function $u_{0}$ is large to the extent of $u_{0}>\eta \uparrow$, the feedback term dominates the leakage initially and its effect tends to grow and eventually leads to the unboundedness of the solution in finite time. It is interseting to note that our estimate indicates that the finite escape time $T_{0}$ is larger for smaller values of $a$ and becomes smaller as $a$ gets larger. In fact, if we increases the value of $a$ from $a \leqq 0$ to $a>0$ then the system (1.1)-(1.3) changes from global asymptotic stability to regional asymptotic stability, and as $a$ increases, the stability region gets smaller while the instability region becomes larger.

It is to be noted that there remains a gap between the stability region $\Lambda_{1}$ and the instability region $\Lambda_{2}$ (by a factor of $\psi_{m}^{-1}$ ). This gap can be closed if one can show that for $u_{0}$ not in these two regions the corresponding solution lies either in $\Lambda_{1}$ or in $\Lambda_{2}$ after a finite time.

\section{Proofs of the main theorems.}

Proof of Theorem 1. We first construct a function $\boldsymbol{u}$ satisfying the reversed inequalities in (2.1). Let $\boldsymbol{u}=e^{-\lambda_{0} t} p(t) \psi(x)$ with $p(0) \leqq b$, where $p(t)$ is a continuously differentiable function to be chosen. Since $\boldsymbol{u}(0, x)=p(0) \psi(x) \leqq u_{0}(x)$ and by $(2.4) \Delta \cdot(D \nabla \boldsymbol{u})=-\lambda_{0} \boldsymbol{u}$ and $\beta \partial \boldsymbol{u} / \partial \nu+\boldsymbol{u}=0$ we see that $\boldsymbol{u}$ is a lower solution if

$$
e^{-\lambda_{0} t} P^{\prime}(t) \psi(x) \leqq a\left(e^{-\lambda_{0} t} P(t) \psi(x)\right)^{1+\alpha},
$$

where $P^{\prime} \equiv d P / d t$. Clearly, the above inequality holds if

$$
\left.P^{\prime} \leqq a_{1}\right\}_{m}^{\alpha} e^{-\alpha \lambda_{0} t} P^{1+\alpha}
$$

It is easily seen that the function $P(t)$ given by

$$
P(t)=\left[b^{-\alpha}+a \lambda_{0}^{-1} \psi_{m}^{\alpha}\left(1-e^{-\alpha \lambda_{0} t}\right)\right]^{-1 / \alpha}, \quad\left(t \in\left[0, T_{1}\right)\right)
$$

satisfies the requirement in (3.2) for $t \in\left[0, T_{1}\right)$, where $T_{1}$ is given by the right side of (2.6). Hence the function $\boldsymbol{u}=e^{-\lambda_{0} t} P(t) \psi(x)$ with $P(t)$ given by (3.3) is a lower solution on $\left[0, T_{2}\right] \times \bar{\Omega}$ for every $T_{2}<T_{1}$. Using this function as the initial iteration in (2.2) with $T<T_{1}$ we obtain a monotone nondecreasing sequence which converges to a solution $u$ of $(1.1)-(1.3)$ on $[0, T] \times \bar{\Omega}$ provided that this sequence is 
uniformly bounded from above. However, this may not be the case for every $T<T_{1}$ unless $u$ has the finite escape time exactly at $T_{1}$. This is due to the fact that $\boldsymbol{u}$ is a lower bound of $u$ when the solution exists. To overcome this difficulty we take an arbitrarily large constant $M>u_{0}(x)$ and define a function $f(u)$ by

$$
f(u)=a u^{1+\alpha} \text { when } u \leqq M \text { and } f(u)=a M^{1+\alpha} \text { when } u>M .
$$

Choose $T_{2}$ sufficiently close to $T_{1}$ such that $\boldsymbol{u}\left(T_{2}, x\right) \geqq M$ for some $x \in \bar{\Omega}$. Then by replacing the right-side of the first equation in (2.2) by $f\left(u^{(h-1)}\right)$ and using the initial iteration $u^{(0)}=\boldsymbol{u}$ with $T=T_{2}$ we again obtain a monotone nondecreasing sequence for the "modified" system (1.1)-(1.3) (i.e., with $a u^{1+\alpha}$ repalced by $f(u)$ ). We denote this sequence also by $\left\{\underline{u}^{(k)}\right\}$. Now since $f\left(\underline{u}^{(k)}\right)$ is uniformly bounded, an application of the well-known estimate for linear parabolic systems implies that $\left\{\underline{u}^{(k)}\right\}$ is bounded (e.g., see [3] p. 146). It follows from the monotone property that $\left\{\underline{u}^{(k)}\right\}$ converges to a unique solution $u^{*}(t, x)$ of the modified system (1.1)-(1.3) and

$$
u^{*}(t, x) \geqq \boldsymbol{u}(t, x) \equiv e^{-\lambda_{0} t} P(t) \psi(x) \quad\left(t \in\left[0, T_{2}\right] \times \bar{\Omega}\right) .
$$

But $\boldsymbol{u}\left(T_{2}, x\right) \geqq M$ for some $x \in \bar{\Omega}$ and $u_{0}(x)<M$, there exists $T_{3} \leqq T_{2}$ such that $u^{*}(t, x) \leqq M$ for $(t, x) \in\left[0, T_{3}\right] \times \bar{\Omega}$ and $u^{*}\left(T_{3}, x\right)=M$ for some $x \in \bar{\Omega}$. By the definition of $f(u), u^{*}$ is the solution of (1.1)-(1.3) with $T=T_{3}$. Since $M$ can be chosen arbitrarily large the above conclusion implies that the solution of (1.1)-(1.3) must be unbounded on $\left[0, T_{0}\right] \times \bar{\Omega}$ for some $T_{0} \leqq T_{1}$. For if it were bounded (say, by $K$ ) on $[0, T] \times \bar{\Omega}$ for every $T \leqq T_{1}$, then by choosing $M>K$ in the definition of $f(u)$ we obtain a solution $u^{*}$ of (1.1)-(1-3) such that $u^{*}(t, x)=M$ at some point $(t, x) \in\left[0, T_{3}\right] \times \bar{\Omega}$ where $T_{3}<T_{1}$. Clearly this is impossible. The proof of the theorem is completed.

Proof of Theorem 2. For the case $a>0$ it suffices to show that $\widetilde{u} \equiv \rho_{\lambda} e^{-\lambda t} \gamma^{\prime}(x)$ is an upper solution on $[0, T] \times \bar{\Omega}$ for every finite $T$. In view of (2.1) and since the last two conditions are satisfied, this will follow if

$$
\left(\lambda_{0}-\lambda\right) \rho_{\lambda} e^{-\lambda t} \psi(x) \geqq a\left(\rho_{\lambda} e^{-\lambda t} \psi(x)\right)^{1+\alpha} .
$$

The above inequality is clearly satisfied for any $\rho_{\lambda}$ satisfying $\rho_{\lambda}^{\alpha} \leqq$ $\left(\lambda_{0}-\lambda\right) / a$. This proves (2.7). When $a \leqq 0$, the sequence obtained from (2.2) with $u^{(0)}=\widetilde{u} \equiv \rho e^{-\lambda t} \gamma^{\prime}(x)$ is no longer monotone nonincreasing and thus we need to modify our construction. To achieve this, we replace the first equation in (2.2) by

$$
\begin{aligned}
u_{t}^{(k)} & -\nabla \cdot\left(D(x) \nabla u^{(k)}\right)+Q u^{(k)} \\
& =a\left(u^{(k-1)}\right)^{1+\alpha}+Q u^{(k-1)}, \quad(t \in(0, T], x \in \Omega)
\end{aligned}
$$


where $Q$ is a constant satisfying $Q \geqq-a \rho^{\alpha}$ while $T<\omega$ is arbitrary. It is easily seen by using the initial iteration $u^{(0)}=\rho e^{-\lambda t} \gamma r(x)$ that the sequence constructed from (3.7) together with the last two equations in (2.2) is nonnegative and is monotone nonincreasing (cf. [10]). Therefore it converges to a unique solution $u(t, x)$ of the system (1.1)-(1.3) such that $0 \leqq(t, x) \leqq \rho e^{-t \lambda} r(x)$ on $[0, T] \times \bar{\Omega}$. This proves the theorem.

\section{REFERENCES}

1. C. Y. Chan, Existence, uniqueness, upper and lower bounds of solutions of nonlinear space-time nuclear reactor kinetics, SIAM J. Appl. Math.. 27 (1974), 72-82.

2. A. Friedman, Remarks on nonlinear parabolic equations, Proc. Amer, Math. Soc.; Symp. Appl. Math., 17 (1965), 3-23.

3. — Partial Differential Equations of Parabolic Typo, Prentice Hall, Englewood Cliffs, N.J., 1964.

4. H. Fujita, On the blowing up of solutions of the Cauchy problem for $u_{t}=\Delta u+$ $u^{1+\alpha}$, J. Fac. Sci. Univ. Tokyo Sect. I, 13 (1966), 109-124.

5. - On some nonexistence and nonuniqueness theorems for nonlinear parabolic equaiions, Proc. Symp. Pure Math. Vol. XVIII, Part 1, Amer. Math. Soc., (1970), 105113.

6. K. Hayakawa, On nonexistence of global solutions of some semi-linear parabolic differential equations, Proc. Japan Acad., 49 (1973), 503-505.

7. S. Kaplan, On the growth of solutions of quasi-linear parabolic equations, Comm. Pure Appl. Math., 16 (1963), 305-330.

8. W.E. Kastenberg and P.L. Chambré, On the stability of nonlinear space-dependent reactor kinetics, Nucl. Sci. Engrg., 31 (1968), 67-79.

9. H. A. Levine and L.E. Payne, Nonexistence of global weak solutions for classes of nonlinear wave and parabolic equations, J. Math. Anal. App., 55 (1976), 329-334.

10. C. V. Pao, Positive solutions of a nonlinear boundary value problem of parabolic tape, J. Differential Equations, 22 (1967), 145-163.

11. - Nonexistence of global solutions and bifurcation analysis for a boundaryvalue problem of parabolic type, Proc. Amer. Math. Soc., (to appear).

12. - Successive approximations of some nonlinear initial boundary value problems, SIAM J. Math. Anal., 46 (1974), 91-102.

13. S.L. Portnoy, On solutions to $u_{t}=\Delta u+u^{2}$ in two dimensions, J. Math. Anal. A.ppl., 55 (1976), 291-294.

14, M. H. Protter and H.F. Weinberger, On the spectrum of general second order operators, Bull. Amer. Math. Soc., 72 (1966), 251-255.

15. G. Rosen, Solutions to the nonlinear recombination equation for the infinite spatial domain, SIAM J. Appl. Math., 29 (1975), 146-151.

16. S. Sugitani. On non-existence of global solutions for some nonlinear integral equations, Osaka J. Math., 12 (1975), 45-51.

Received March 4, 1977.

North Carolina State University

RALEIGH, NC 27650

AND

UNIVERSITÄT GRAZ

Graz, Austria 



\section{PACIFIC JOURNAL OF MATHEMATICS}

\section{EDITORS}

DONALD BABBITT (Managing Editor)

University of California

Los Angeles, California 90024

Hugo RossI

University of Utah

Salt Lake City, UT 84112

C. C. MOORE and ANDrew OGG

University of California

Berkeley, CA 94720
J. DUgundjI

Department of Mathematics University of Southern California Los Angeles, California 90007

R. FinN aNd J. Milgram Stanford University Stanford, California 94305

\section{ASSOCIATE EDITORS}

E. F. BECKENBACH

B. H. NeUMANN

F. WOLF

K. YosHIDA

\section{SUPPORTING INSTITUTIONS}

UNIVERSITY OF BRITISH COLUMBIA CALIFORNIA INSTITUTE OF TECHNOLOGY UNIVERSITY OF CALIFORNIA MONTANA STATE UNIVERSITY UNIVERSITY OF NEVADA, RENO NEW MEXICO STATE UNIVERSITY OREGON STATE UNIVERSITY UNIVERSITY OF OREGON
UNIVERSITY OF SOUTHERN CALIFORNIA STANFORD UNIVERSITY UNIVERSITY OF HAWAII UNIVERSITY OF TOKYO UNIVERSITY OF UTAH WASHINGTON STATE UNIVERSITY UNIVERSITY OF WASHINGTON 


\section{Pacific Journal of Mathematics}

\section{Vol. 84 , No. 1 \\ May, 1979}

Michael James Beeson, Goodman's theorem and beyond ...............

Robert S. Cahn and Michael E. Taylor, Asymptotic behavior of multiplicities

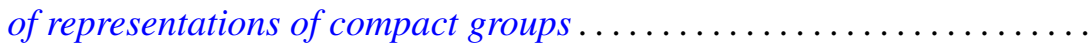

Douglas Michael Campbell and Vikramaditya Singh, Valence properties of

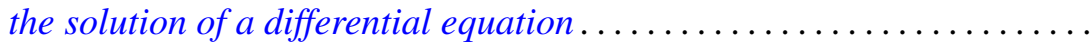

J.-F. Colombeau, Reinhold Meise and Bernard Perrot, A density result in spaces of Silva holomorphic mappings .....................

Marcel Erné, On the relativization of chain topologies .................

Le Baron O. Ferguson, Uniform and $L_{p}$ approximation for generalized

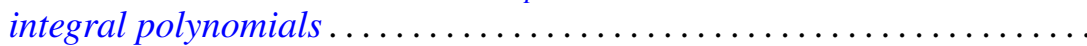

Kenneth R. Goodearl and David E. Handelman, Homogenization of regular rings of bounded index..................................

Friedrich Haslinger, A dual relationship between generalized

Abel-Gončarov bases and certain Pincherle bases .................

Miriam Hausman, Generalization of a theorem of Landau. .

Makoto Hayashi, 2-factorization in finite groups

Robert Marcus, Stochastic diffusion on an unbounded domain ........... 143

Isabel Dotti de Miatello, Extension of actions on Stiefel manifolds....

C. David (Carl) Minda, The hyperbolic metric and coverings of Riemann surfaces...

Somashekhar Amrith Naimpally and Mohan Lal Tikoo, On $T_{1}$-compactifications.

Chia-Ven Pao, Asymptotic stability and nonexistence of global solution for a semilinear parabolic equation ....

Shigeo Segawa, Harmonic majoration of quasibounded type ...

Sze-Kai Tsui and Steve Wright, The splitting of operator algebras ...

Bruce Williams, Hopf invariants, localization and embeddings of Poincaré complexes....

Leslie Wilson, Nonopenness of the set of Thom-Boardman maps ...

Alicia B. Winslow, There are $2^{\mathrm{c}}$ nonhomeomorphic continua in $\beta R^{n}-R^{n}$ 